\title{
Automation of one-loop scattering amplitudes with Golem/Samurai
}

\section{G. Cullen ${ }^{1}$, N. Greiner ${ }^{2,3}$, G. Heinrich ${ }^{2}$, G. Luisoni ${ }^{4}$, P. Mastrolia ${ }^{2}$, G. Ossola ${ }^{*, 5,6}$,} T. Reiter ${ }^{2}$, F. Tramontano ${ }^{7}$

${ }^{1}$ School of Physics and Astronomy, The University of Edinburgh, Edinburgh EH9 3JZ, UK

${ }^{2}$ Max-Planck Insitut für Physik, Föhringer Ring, 6, D-80805 München, Germany

${ }^{3}$ Department of Physics, University of Illinois at Urbana-Champaign, $1110 \mathrm{~W}$ Green Street, Urbana IL 61801, USA

${ }^{4}$ IPPP, University of Durham, Durham DH1 3LE, UK

5 Physics Department, New York City College of Technology, City University of New York, 300 Jay Street, Brooklyn NY 11201, USA

${ }^{6}$ The Graduate School and University Center, The City University of New York 365 Fifth Avenue, New York NY 10016, USA

7 Theory Group, Physics Department, CERN, 1211 Geneva 23, Switzerland

\begin{abstract}
After a brief review of the various approaches available for the automated computation of multiparticle scattering amplitudes at the one-loop level, in this presentation we describe the main features of the GoSAM (Golem/Samurai) framework. The GoSAM approach combines a ddimensional extension of the integrand-level reduction method and improved tensorial techniques, with an automated generation of amplitudes via Feynman diagrams. GoSAM can be used to generate and evaluate one-loop corrections in both QCD and electro-weak theory, and offers the flexibility to link general model files for theories Beyond the Standard Model. The discussion will be accompanied with examples of applications.
\end{abstract}

The 2011 Europhysics Conference on High Energy Physics-HEP 2011,

July 21-27, 2011

Grenoble, Rhône-Alpes France

\footnotetext{
* Speaker.
} 


\section{Introduction and General Motivations}

In the last few years we observed a tremendous progress in the field of NLO QCD calculations $[1,2]$. The continuous improvement of new techniques for one-loop computations led to several new results for processes with four or more particles in the final state [3-5].

Calculations of increasing complexity have been performed with improved algebraic reduction methods based on Feynman-diagrammatic algorithms, as well as with new numerical techniques based on the idea of reconstructing one-loop amplitudes from their unitarity cuts. These theoretical developments found an ideal counterpart in the integrand-level reduction algorithm, known as OPP method, that allows for the reduction of any scattering amplitudes to scalar master integrals, simply by evaluating numerically the integrand at given fixed values of the integration momentum. In both scenarios, automation is indispensable for processes with many external legs, because of the increase in the complexity and number of diagrams that contribute to the amplitudes.

In this presentation, we illustrate the main features of GoSAM (Golem/Samurai), a new framework for the evaluation of one-loop scattering amplitudes. GoSAM combines the automated algebraic generation of $d$-dimensional unintegrated amplitudes obtained via Feynman diagrams, with the numerical integrand-level reduction provided by the $d$-dimensional extension [6-8] of the OPP integrand-level reduction method [9] and improved tensorial techniques [10,11]. The integrand is generated via Feynman diagrams, using QGRAF [12], FORM [13], spinney [14] and haggies [15]. The individual program tasks are managed by python scripts. The only task required from the user is the preparation of an "input card" in order to launch the generation of the source code and its compilation, without having to worry about internal details of the code generation. A detailed description of the framework, together with all features available in GoSAM, can be found in Ref. [16].

\section{Algebraic approach to Automation}

There are several approaches to the automated computation of multi-particle scattering amplitudes at the one-loop level, which provide different recipes for the construction of multi-purpose tools. The goal of such tools is the evaluation of one-loop scattering amplitudes for any choice of particles in the initial and final states, in a fully automated manner.

At the tree-level, several tools have achieved this task successfully. Important work has been done in the past few years to extend this kind of machinery to the automation of one-loop calculations. In the approach of HELAC-NLO [17] and MadLoop [18], existing codes for the automated evaluation of the tree-level amplitudes [19] have been cleverly extended to compute the one-loop virtual corrections, in combination with the reduction code CutTools [20]. Both codes are fully integrated with real radiation and subtraction terms [21].

As a different approach to multi-purpose automation, amplitudes can be generated from Feynman diagrams by employing tools for algebraic manipulation that have been available for many years, such as QGRAF [12] or FORM [13]. This algebraic generation of diagrams can be combined withe the newly developed integrand-level reduction methods. In this case however the algebraic operations required are quite different with respect to a traditional tensorial reduction: the input of an integrand-level reduction is the numerical value of the numerator function at given values of 
integration momentum. We should aim at compact expressions that provide the numerical value for the unintegrated numerator. To achieve this task, for example, expressions in terms of spinor products are particularly convenient.

Here are some of the advantages of the "algebraic approach": i) algebraic manipulations are allowed before starting the numerical integration, namely the algebraic generation is executed separately from the numerical reduction; CPU-time can be spent, once for all at the beginning of the calculation, to optimize and reduce the size of the integrands that will be evaluated numerically several times later on during the reduction; ii) we can try different optimizations by grouping sets of diagrams and caching in smart ways all factors that do not depend on the integration momentum; iii) we have easy access to sub-parts of the computation, namely subsets of diagrams can be easily moved in/out from the results, to allow for comparisons and tests; iv) we can perform computer algebra in dimension $d$, employing different regularization schemes; v) there is flexibility in the choice of the reduction techniques: the choice between different reduction algorithms can be performed at run-time.

In the next section we will illustrate how these properties are used within GoSam. Important progress in a similar direction has been also achieved within FeynArts/FormCalc/LoopTools [22] to provide amplitudes that can be processed with traditional Passarino-Veltman reduction [23] or using the integrand-level reduction provided by CutTools [20] and/or SAMURAI [8].

\section{A brief introduction to GoSAM}

GoSAM produces in a fully automated way all the code required to perform the calculation of virtual one-loop amplitudes. The user should only prepare an "input card", that contains all the specific information about the process (i.e. initial and final particles, model, helicities, selection rules to exclude particular sets of diagrams, regularization scheme).

The main steps in this process are: the generation of contributing diagrams, the optimization and algebraic manipulation to simplify their expressions, and the writing of a FORTRAN code ready to be used within a phase-space integration. The reduction of unintegrated amplitudes to linear combinations of scalar (master) integrals is fully embedded in the process and can be performed with different options, all available at run-time.

Diagram Generation For the diagram generation both at tree level and one-loop level we employ QGRAF [12]. In addition, we added another filter over diagrams by means of Python. This gives several advantages since it increases the ability of the code to distinguish certain classes of diagrams and group them according to the sets of their propagators, in order to fully optimize the reduction.

At this stage GoSAm generates three sets of output files: an expression for each diagram for FORM [13], Python code for drawing each diagram, and Python code for computing the properties of the diagram. Information about the model is either read from the built-in Standard Model of QGRAF or can be defined by the user by means of LanHEP [24] or an UFO [25] file.

The Python program automatically performs several operations: diagrams whose color factor turns out to be zero are dropped; the number of propagators containing the loop momentum, the tensor rank and the kinematic invariants of the associated loop integral are computed; diagrams with a vanishing loop integral associated are detected and flagged for the diagram selection; 
all propagators and vertices are classified for the diagram selection; diagrams containing massive quark self-energy insertions or closed massless quark loops are specially flagged.

Lorentz Algebra Concerning the algebraic operations performed by GoSAM to render the integral suitable for efficient numerical evaluation, one of the primary goals is to split the $(4-2 \varepsilon)$ dimensional algebra into strictly four-dimensional objects and symbols representing the higherdimensional remainder. All external vectors (momenta and polarisation vectors) are kept in four dimensions; internal vectors, however, are kept in the $d$-dimensional vector space. Details about the conventions used can be found in [14].

Once all propagators and all vertices have been replaced by their corresponding expressions, according to the model file, all vector-like quantities and metric tensors are split into their fourdimensional and their orthogonal part. While the $(d-4)$-dimensional traces are reduced completely to products of $(d-4)$-dimensional metric tensors, the four-dimensional part, that will be reduced numerically, is treated such that the number of terms in the resulting expression is kept as small as possible.

Treatment of rational terms $R_{2}$ Instead of relying on the construction of $R_{2}$ from specialized Feynman rules [26], we can generate the $R_{2}$ part along with all other contribution using automated algebraic manipulations. The code offers the option between the implicit and explicit construction of the $R_{2}$ terms. The implicit construction treats the $4-$ and $(d-4)$ dimensional numerators on equal grounds: they are generated algebraically and reduced numerically. The explicit construction of $R_{2}$ is based on the fact that the $(d-4)$ dimensional part of the numerator function contains expressions for the corresponding integrals that are relatively simple and known explicitly. Therefore, after separating it using the algebraic manipulation described before, the $(d-4)$ dimensional part is computed analytically whereas the purely four-dimensional part is passed to the numerical reduction. This approach also allows for an efficient calculation of the $R_{2}$ alone.

Reduction to scalar (master) integrals GoSAM allows to choose at run-time (namely without regenerating the code) the preferred method of reduction. Available options include the integrallevel $d$-dimensional reduction, as implemented in SAMURAI, or traditional tensor reduction as implemented in Golem95C interfaced through tensorial reconstruction at the integrand level, or a combination of both. Concerning the scalar (tensorial) integrals, GoSAM allows to choose among a variety of options, including QCDLoop [27], OneLoop [28], Golem95C [29], plus the recently added P JFRY [30]. For details about the reduction methods, we refer the reader to previous presentations $[11,31]$ or the original articles.

\section{Examples of Applications}

The BLHA interface [32] allows to link GoSAm to a general Monte Carlo event generator, which is responsible for supplying the missing ingredients for a complete NLO calculation of a physical cross section. Among those, SHERPA [33] offers the possibility to compute the LO cross section and the real corrections with both the subtraction terms and the corresponding integrated counterparts [34]. Using the BLHA interface, we linked GoSAM with SHERPA to compute the physical cross section for $W^{ \pm}+1$-jet at NLO [16]. 
The codes produced by GoSAM have been tested on several processes, some of which are depicted in Table 1. The full list of processes is given in Ref. [16].

\begin{tabular}{|l|l|}
\hline Process & Checked with Ref. \\
\hline$u \bar{d} \rightarrow e^{-} \bar{v}_{e} g$ & {$[18]$} \\
$e^{+} e^{-} \rightarrow e^{+} e^{-} \gamma(\mathrm{QED})$ & {$[36]$} \\
$p p \rightarrow H t \bar{t}$ & {$[18]$} \\
$p p \rightarrow W^{+} W^{+} j j$ & {$[4]$} \\
$p p \rightarrow b \bar{b} b \bar{b}$ & {$[5,37]$} \\
$p p \rightarrow W^{+} W^{-} b \bar{b}$ & {$[18,37]$} \\
$u \bar{u} \rightarrow t \bar{t} b \bar{b}$ & {$[18,37]$} \\
$g g \rightarrow t \bar{t} b \bar{b}$ & {$[18,37]$} \\
$u \bar{d} \rightarrow W^{+} g g g$ & {$[37]$} \\
\hline
\end{tabular}

Table 1: Some of the processes computed and checked with GoSAM

As an example of the usage of GoS AM with a model file different from the Standard Model, we calculated the QCD corrections to neutralino pair production in the MSSM. The model file has been imported using the UFO interface. In this calculation, we combined the one-loop amplitude with the real radiation corrections to obtain results for differential cross sections. For the infrared subtraction terms we employed MadDipole [38], while the real emission part is calculated using MadGraph/MadEvent [39].

\section{Conclusions and Outlook}

Several groups are currently working at the development of automated multi-purpose tools for one-loop calculations. Following the example of tree-level multi-process tools, already developed some time ago, the current target is to build, at the one-loop level, efficient and flexible programs that can be used to tackle the increasing need of precision required by the experimental analysis at the LHC.

Aside from improvements on standard tensorial techniques, the progress on unitarity-based approaches, combined with the reduction at the integrand level, opened the possibility for the development of new numerical and semi-algebraic approaches for one-loop calculations. Moreover, we already witnessed preliminary attempts to generalize and extend these methods to the case of higher-order calculations [40].

In this presentation, we described the main features of GoSAM [16], a flexible and broadly applicable tool for the fully automated evaluation of one-loop scattering amplitudes. The amplitudes are generated in terms of Feynman diagrams and the reduction to master integrals can be performed in several ways, which can be selected at run-time. The code performed well in reproducing a wide series of examples, and it is ready to be applied to more challenging calculations.

\section{Acknowledgments}

G.C. and G.L. are supported by the British Science and Technology Facilities Council (STFC). N.G. was supported in part by the U.S. Department of Energy under contract No. DE-FG02-91ER40677. P.M. and T.R. were supported by the Alexander von Humboldt Foundation, in the framework of the Sofja Kovaleskaja 
Award Project "Advanced Mathematical Methods for Particle Physics", endowed by the German Federal Ministry of Education and Research. The work of G.O. was supported in part by the National Science Foundation under Grant PHY-0855489. G.O. also acknowledges the support of the Center for Theoretical Physics at City Tech. The research of F.T. is supported by Marie-Curie-EIF, project: "SAMURAI-Apps".

\section{References}

[1] Z. Bern et al. arXiv:0803.0494 [hep-ph]; J. R. Andersen et al. arXiv:1003.1241 [hep-ph].

[2] Z. Bern, L. J. Dixon and D. A. Kosower, Annals Phys. 322 (2007) 1587;

R. K. Ellis, Z. Kunszt, K. Melnikov and G. Zanderighi, arXiv:1105.4319 [hep-ph].

[3] C. F. Berger et al., Phys. Rev. D80 (2009) 074036; C. F. Berger et al., Phys. Rev. Lett. 102 (2009) 222001; R. K. Ellis, K. Melnikov, G. Zanderighi, Phys. Rev. D80 (2009) 094002; K. Melnikov, G. Zanderighi, Phys. Rev. D81 (2010) 074025; C. F. Berger et al., Phys. Rev. D82 (2010) 074002; A. Bredenstein et al., Phys. Rev. Lett. 103 (2009) 012002; A. Bredenstein et al., JHEP 1003 (2010) 021; G. Bevilacqua et al., JHEP 0909 (2009) 109; G. Bevilacqua et al., Phys. Rev. Lett. 104 (2010) 162002; G. Bevilacqua et al., JHEP 1102 (2011) 083; G. Bevilacqua et al., [arXiv:1108.2851 [hep-ph]]; A. Denner et al., Phys. Rev. Lett. 106 (2011) 052001; T. Melia et al., Phys. Rev. D83 (2011) 114043; F. Campanario et al., Phys. Lett. B704 (2011) 515-519; C. F. Berger et al., Phys. Rev. Lett. 106 (2011) 092001; H. Ita et al., [arXiv:1108.2229 [hep-ph]].

[4] T. Melia et al., JHEP 1012 (2010) 053.

[5] T. Binoth et al., Phys. Lett. $B 685$ (2010) 293-296;

N. Greiner et al., Phys. Rev. Lett. 107 (2011) 102002.

[6] R. K. Ellis et al., Nucl. Phys. B822 (2009) 270-282.

[7] P. Mastrolia et al., JHEP 0806 (2008) 030.

[8] P. Mastrolia et al., JHEP 1008 (2010) 080.

[9] G. Ossola, C. G. Papadopoulos, R. Pittau, Nucl. Phys. B763 (2007) 147-169;

G. Ossola, C. G. Papadopoulos, R. Pittau, JHEP 0707 (2007) 085;

G. Ossola, C. G. Papadopoulos, R. Pittau, JHEP 0805 (2008) 004.

[10] G. Heinrich et al., JHEP 1010 (2010) 105.

[11] T. Reiter et al., [arXiv:1011.6632 [hep-ph]].

[12] P. Nogueira, J. Comput. Phys. 105 (1993) 279-289.

[13] J. A. M. Vermaseren, arXiv:math-ph/0010025.

[14] G. Cullen, M. Koch-Janusz, T. Reiter, Comput. Phys. Commun. 182 (2011) 2368-2387.

[15] T. Reiter, Comput. Phys. Commun. 181, 1301 (2010).

[16] G. Cullen et al., arXiv:1111.2034 [hep-ph].

[17] G. Bevilacqua et al., [arXiv:1110.1499 [hep-ph]].

[18] V. Hirschi et al., JHEP 1105 (2011) 044.

[19] A. Kanaki and C. G. Papadopoulos, Comput. Phys. Commun. 132 (2000) 306; A. Cafarella, C. G. Papadopoulos and M. Worek, Comput. Phys. Commun. 180, 1941 (2009); T. Stelzer and W. F. Long, Comput. Phys. Commun. 81 (1994) 357. 
[20] G. Ossola, C. G. Papadopoulos, R. Pittau, JHEP 0803 (2008) 042.

[21] M. Czakon, C. G. Papadopoulos and M. Worek, JHEP 0908, 085 (2009);

R. Frederix, S. Frixione, F. Maltoni and T. Stelzer, JHEP 0910, 003 (2009).

[22] T. Hahn, M. Perez-Victoria, Comput. Phys. Commun. 118 (1999) 153-165;

T. Hahn, Comput. Phys. Commun. 140 (2001) 418-431; T. Hahn, PoS ACAT2010 (2010) 078;

S. Agrawal, T. Hahn and E. Mirabella, arXiv:1112.0124 [hep-ph].

[23] G. 't Hooft and M. J. G. Veltman, Nucl. Phys. B 153 (1979) 365;

G. Passarino and M. J. G. Veltman, Nucl. Phys. B 160 (1979) 151.

[24] A. Semenov, [arXiv:1005.1909 [hep-ph]].

[25] C. Degrande et al., [arXiv:1108.2040 [hep-ph]].

[26] P. Draggiotis et al., JHEP 0904 (2009) 072; M. V. Garzelli, I. Malamos, R. Pittau, JHEP 1001 (2010) 040; M. V. Garzelli, I. Malamos, R. Pittau, JHEP 1101 (2011) 029; M. V. Garzelli, I. Malamos, Eur. Phys. J. C71 (2011) 1605.

[27] R. K. Ellis, G. Zanderighi, JHEP 0802 (2008) 002;

G. J. van Oldenborgh, Comput. Phys. Commun. 66 (1991) 1-15.

[28] A. van Hameren, Comput. Phys. Commun. 182 (2011) 2427-2438.

[29] T. Binoth et al., Comput. Phys. Commun. 180 (2009) 2317-2330;

G. Cullen et al., Comput. Phys. Commun. 182 (2011) 2276-2284.

[30] J. Fleischer, T. Riemann and V. Yundin, arXiv:1112.0500 [hep-ph].

[31] G. Ossola, PoS ACAT2010 (2010) 075;

G. Cullen et al., Nucl. Phys. Proc. Suppl. 205-206 (2010) 67-73.

[32] T. Binoth et al., Comput. Phys. Commun. 181 (2010) 1612-1622.

[33] T. Gleisberg et al., JHEP 0902 (2009) 007.

[34] F. Krauss, R. Kuhn, G. Soff, JHEP 0202 (2002) 044; M. Schonherr, F. Krauss, JHEP 0812 (2008) 018.

[35] T. Gleisberg, F. Krauss, Eur. Phys. J. C53 (2008) 501-523.

[36] S. Actis, P. Mastrolia, G. Ossola, Phys. Lett. B682 (2010) 419-427;

S. Actis, P. Mastrolia, G. Ossola, Acta Phys. Polon. B40 (2009) 2957-2963.

[37] A. van Hameren, C. G. Papadopoulos, R. Pittau, JHEP 0909 (2009) 106.

[38] R. Frederix, T. Gehrmann, N. Greiner, JHEP 0809 (2008) 122;

R. Frederix, T. Gehrmann, N. Greiner, JHEP 1006 (2010) 086.

[39] J. Alwall et al., JHEP 0709 (2007) 028.

[40] J. Gluza, K. Kajda, D. A. Kosower, Phys. Rev. D83 (2011) 045012;

P. Mastrolia, G. Ossola, [arXiv:1107.6041 [hep-ph]];

D. A. Kosower, K. J. Larsen, [arXiv:1108.1180 [hep-th]]. 